\title{
FATORES PROGNÓSTICOS DO TRAUMA RAQUIMEDULAR POR PROJETIL DE ARMA DE FOGO EM PACIENTES SUBMETIDOS A LAMINECTOMIA
}

\author{
LEANDRO PRETTO FLORES* JOÃO DE SOUSA NASCIMENTO FILHO**, \\ ALDO PEREIRA NETO***, KUNIO SUZUKI****
}

\begin{abstract}
RESUMO: Os traumas sobre a coluna resultantes de projetil de arma de fogo (PAF) são lesões geralmente graves e muitas vezes com baixo potencial para recuperação neurológica. A indicação cirúrgica destas lesões ainda é motivo de controvérsia. O objetivo deste artigo é identificar fatores no pré e trans-operatório que irão influenciar na recuperação neurológica destes pacientes. Realizamos estudo retrospectivo de 45 pacientes submetidos a laminectomia para trauma raquimedular por PAF, avaliando os seguintes fatores: nível da lesão, apresentação clínica, uso de glicocorticóide no pré-operatório, presença de lesão dural, momento cirúrgico e relação entre escala de Frankel pré e pós-operatória. Observamos que os fatores mais importantes para a recuperação neurológica foram o nível da lesão (53\% dos pacientes com lesão lombar melhoraram após a cirurgia) e a apresentação clínica pré-operatória (pacientes com síndrome de cauda equina obtiveram melhora em $60 \%$ dos casos), sendo que as demais variáveis não apresentaram significância estatística. Em $71 \%$ dos casos, a dor pré-operatória foi aliviada com o procedimento cirúrgico.
\end{abstract}

PALAVRAS-CHAVE: trauma raquimedular, projetil de arma de fogo, prognóstico.

\section{Prognostic factors related to gunshot wounds to the spine in patients submited to laminectomy}

ABSTRACT - The spinal trauma related to civilian gunshot missile still remains a serious neurological event that carries a dismal prognosis almost in all cases. Its surgical indication also is a mather of discution. Our goal is to identify the aspects that could influence the prognosis after surgery to this kind of lesions. We conducted a retrospective study of 45 consecutive patients submitted to laminectomy at Hospital de Base do Distrito Federal (Brasília, Brazil), testing the following aspects: initial neurological status, level of the deficit, surgical timing, use of methilprednisolone and presence of dural tearing. Among those, the initial clinical presentation and the level of the lesion $(60 \%$ of the patients with cauda equina syndrome and $53 \%$ of that with lesions in the lombar region improved their neurological status after laminectomy) were the most important factors affecting the outcome. Seventy percent of the patients experienced a pain relief after the surgical procedure.

KEY-WORDS: spinal trauma, gunshot wound, prognosis.

O traumatismo raquimedular (TRM) penetrante, seja ele provocado por projetil de arma de fogo (PAF) ou objetos perfurantes constitui lesão grave e geralmente associada a mau prognóstico neurológico ${ }^{1}$. Quando um projetil atinge a coluna vertebral provoca lesões primárias devido ao trauma mecânico direto e trauma térmico sobre as estruturas neurais e vasculares. Posteriormente pode desencadear lesões secundárias, tanto por alterações isquêmicas como hiperêmicas sobre o

Unidade de Neurocirurgia do Hospital de Base do Distrito Federal, Brasília, DF: *Residente em Neurocirurgia; **Médico Ortopedista; $* * *$ Staff Neurocirurgião; ****Chefe da Unidade de Neurocirurgia do HBDF. Aceite: 14-junho-1999.

Dr. Leandro Pretto Flores - SHCGN 716 Bloco J Apartamento 119 Asa Norte - 70770-740 Brasília DF - Brasil.

E-mail: ifneiva@nutecnet.com.br 
tecido lesado ${ }^{2,3}$. Estes mecanismos provocam lesões muitas vezes definitivas sobre os elementos nervosos, justificando o mau prognóstico observado nestes doentes.

Ainda hoje existe controvérsia quanto ao papel da cirurgia como tratamento para esse tipo de lesão, principalmente porque estudos recentes demonstram que o prognóstico desses doentes não é diferente quando tratados conservadoramente ${ }^{4-8}$. Ainda assim, a cirurgia continua tendo seu lugar na terapêutica, principalmente nos pacientes que apresentam déficits em progressão ou quando os exames de imagem mostram que existe compressão sobre as estruturas neurovasculares ${ }^{2,9}$. A principal abordagem nestes casos é a laminectomia, tendo como objetivo a descompressão do canal medular ${ }^{7,8}$; em alguns outros casos a fixação de uma coluna instável ${ }^{10}$ ou a descompressão radicular para alívio da dor ${ }^{11}$ também são procedimentos utilizados.

A indicação cirúrgica, portanto, deve ser sempre individualizada. A determinação de fatores observados no pré-operatório que possam influenciar no prognóstico desses pacientes é útil ao neurocirurgião para auxiliar na decisão cirúrgica. O objetivo deste estudo é avaliar os fatores no pré e trans-operatório que possam influenciar o curso dos pacientes submetidos a laminectomia devido a agressão por arma de fogo sobre a coluna, além de discutir o controle cirúrgico da dor nestes pacientes.

\section{MÉTODO}

Foi realizado estudo retrospectivo, através da análise dos prontuários dos pacientes submetidos a laminectomia como tratamento para TRM por PAF, na Unidade de Neurocirurgia do Hospital de Base do Distrito Federal (HBDF), durante o período de 1990-1998, totalizando 45 pacientes. Foram colhidos dados quanto ao sexo, idade, nível da lesão, apresentação clínica, classificação na escala de Frankel ${ }^{12}$ pré e pós-operatória, presença de laceração dural, síntese ou não da dura-mater, momento cirúrgico, complicações pós-operatórias e uso ou não de antibióticos e/ou glicocorticóides. Os pacientes foram divididos quanto à apresentação clínica, em lesões completas (Frankel A), lesões incompletas (Frankel B,C, ou D em lesões medulares) e lesões de cauda equina (Fig 1). Como critério de melhora da função neurológica, foi considerada qualquer melhora, de acordo com a escala de Frankel, no seguimento pós-operatório. O alívio da dor foi considerado de acordo com a sensação de melhora subjetiva relatada pelo paciente.

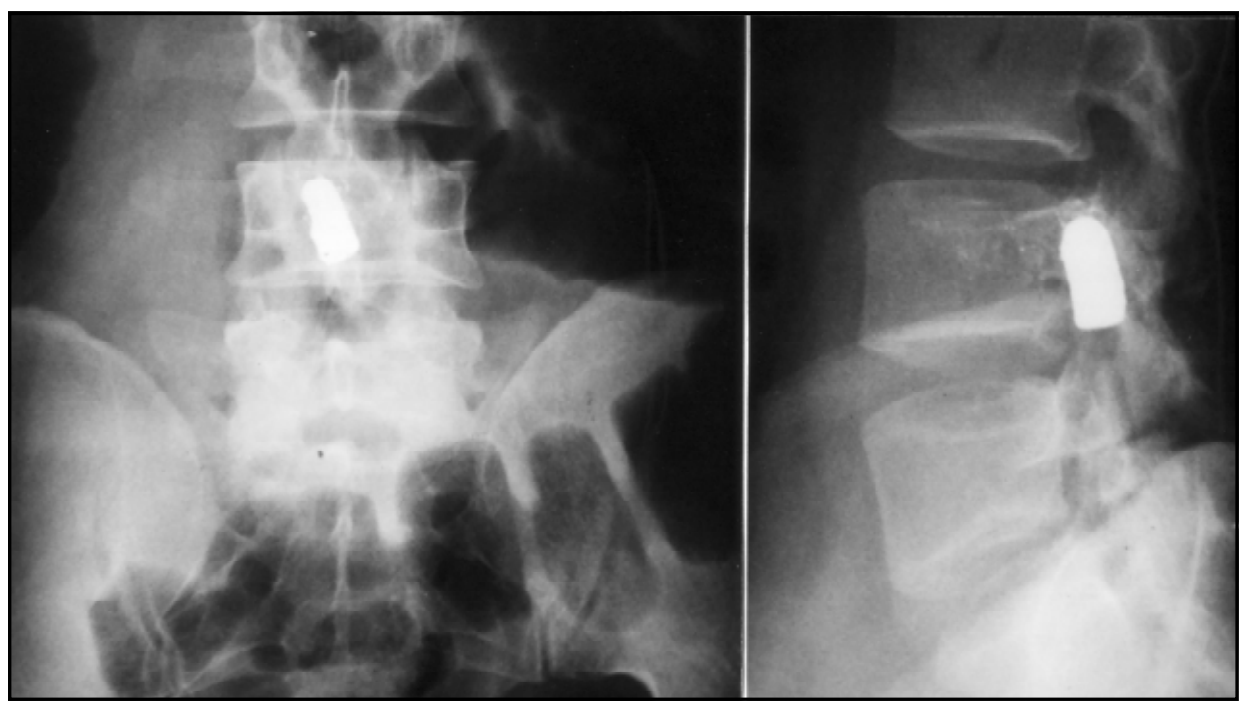

Fig 1. Paciente de 24 anos, vítima de agressão por arma de fogo em região lombar. Deu entrada no ProntoSocorro com paraparesia assimétrica, Frankel C. Raio X de coluna lombar em incidência antero-posterior (esquerda) e perfil (direita) mostrando projetil no interior do canal a nível L4. Após a cirurgia, evoluiu com recuperação complera do déficit, Frankel E. 
O tempo de seguimento variou de quatro a 36 meses, com uma média de 19 meses. A análise estatística foi realizada utilizando-se o teste do chi-quadrado, tendo como significantes valores com $\mathrm{p}<0,05$.

\section{RESULTADOS}

Dos 45 pacientes estudados, 38 eram do sexo masculino e sete do sexo feminino. A maioria dos pacientes encontrava-se com idade entre a segunda e terceira décadas (86\%) e o principal nível da lesão foi a região lombar (oito pacientes com lesão cervical, onze lesões torácicas e 26 lombares). Em apenas cinco doentes o projetil penetrou inicialmente a cavidade torácica ou abdominal antes de atingir a coluna. Secção completa da medula ocorreu em 15 pacientes (33\%), secção incompleta em $12(27 \%)$, em 15 doentes encontramos síndrome da cauda equina (33\%) e três pacientes não apresentavam déficits à admissão. Dor pré-operatória foi referida por 14 pacientes (31\%), sendo que em 9 (20\%) esta era caracterizada como de padrão radicular, em 4 (9\%) a dor era local ( por lesão óssea ou de partes moles) e um paciente queixava-se de disestesias importantes nos membros inferiores, possivelmente devido a lesão do trato espinho-talâmico lateral. Em três casos (6\%) observava-se fístula liquórica antes do procedimento cirúrgico. Todos os pacientes fizeram uso de antibióticos por período mínimo de dez dias (oxacilina, cloranfenicol, ampicilina ou cefazolina, em diferentes combinações), e em 32 pacientes (71\%) foi utilizado glicocorticóide no período préoperatório (metilprednisolona, $30 \mathrm{mg} / \mathrm{kg}$ em dose de ataque e manutenção por $23 \mathrm{hs}$ com dose de 5,4 $\mathrm{mg} / \mathrm{kg} / \mathrm{h}$, nos casos admitidos com menos de 8 horas após a agressão). Quanto ao momento cirúrgico, os pacientes foram divididos entre aqueles operados com menos de seis horas (nove casos), entre 612 horas (oito casos), 12-24 horas (oito pacientes) e com mais de 24 horas após a agressão (20 pacientes). Estes grupos representam uma divisão rotineira do nosso serviço para avaliação de disponibilidade de centro cirúrgico para neurotrauma quando a emergência não é o principal critério. A lesão dural foi identificada em 25 casos (55\%), sendo possível a sutura primária em 15, e, nos restantes, a dura foi deixada aberta.

A Tabela 1 relaciona a distribuição dos pacientes de acordo com a escala de Frankel no período pré e pós-operatório, além de distribuí-los também de acordo com o nível da lesão encontrado. Observa-se que a maioria dos pacientes com lesão em região lombar apresentou, à admissão, escores entre C e E na escala de Frankel, havendo melhora funcional em 53\%. Já os pacientes com trauma nas regiões torácica e cervical apresentaram-se, geralmente, com déficits iniciais mais importantes (Frankel A ou B). Ferimentos localizados na região torácica apresentaram os menores graus de

Tabela 1. Distribuição dos pacientes de acordo com o nível da lesão, relacionando com a escala de Frankel no período pré e pós-operatório, em 45 pacientes com trauma raquimedular por projetil de arma de fogo, submetidos a laminectomia.

\begin{tabular}{llccccc}
\hline \multirow{2}{*}{ Lombar } & & A & B & Crankel & D & E \\
\hline \multirow{3}{*}{ Torácico } & Pré-op. & 5 & 5 & 5 & 8 & 3 \\
& Pós-op. & 3 & 2 & 5 & 8 & 8 \\
& Pré-op. & 7 & 2 & 1 & 1 & 0 \\
\multirow{2}{*}{ Cervical } & Pós-op. & 6 & 2 & 1 & 1 & 1 \\
& Pré-op. & 3 & 3 & 1 & 1 & 0 \\
& Pós-op. & 3 & 0 & 3 & 2 & 0 \\
& Pré-op. & 15 & 10 & 7 & 10 & 3 \\
& Pós-op. & 12 & 4 & 9 & 11 & 9 \\
\hline
\end{tabular}


Tabela 2. Valor prognóstico de diferentes variáveis em estudo em 45 pacientes com trauma raquimedular por projetil de arma de fogo, submetidos a laminectomia.

\begin{tabular}{|c|c|c|c|c|}
\hline \multicolumn{2}{|l|}{ Variável } & \multirow{3}{*}{$\begin{array}{c}\begin{array}{c}\text { pacientes } \\
(n=45) \\
\mathrm{n}\end{array} \\
26\end{array}$} & \multirow{3}{*}{$\begin{array}{c}\text { pacientes } \\
\text { que melhoraram } \\
\mathrm{n}(\%) \\
14(53)\end{array}$} & \multirow{3}{*}{$\begin{array}{c}\mathrm{p} \\
\\
<0,05\end{array}$} \\
\hline & & & & \\
\hline -Nível & Lombar & & & \\
\hline & Torácico & 11 & $4(36)$ & $<0,05$ \\
\hline & Cervical & 8 & $4(50)$ & $<0,05$ \\
\hline \multirow[t]{3}{*}{-Clínica } & Secção completa & 15 & $3(20)$ & $<0,05$ \\
\hline & Secção incompleta & 12 & $6(50)$ & $<0,05$ \\
\hline & Cauda eqüina & 15 & $9(60)$ & $<0,05$ \\
\hline \multirow[t]{2}{*}{-Corticóide } & Sim & 32 & $18(50)$ & NS \\
\hline & Não & 13 & $4(30)$ & NS \\
\hline \multirow[t]{4}{*}{-“Timming" Cirúrgico } & $0-6 \mathrm{~h}$ & 9 & $4(44)$ & NS \\
\hline & $6-12 \mathrm{~h}$ & 8 & $5(62)$ & NS \\
\hline & $12-24 \mathrm{~h}$ & 8 & $3(37)$ & NS \\
\hline & $>24 \mathrm{~h}$ & 20 & $10(50)$ & NS \\
\hline \multirow[t]{2}{*}{-Lesão dural } & Sim & 25 & $12(48)$ & NS \\
\hline & Não & 20 & $10(50)$ & NS \\
\hline
\end{tabular}

NS, não significativo

recuperação neurológica, ocorrendo melhora em apenas 36\% dos casos: um paciente evoluiu de Frankel A para B, um de D para E, um de C para D e um caso com evolução de B para C.

Quanto aos resultados cirúrgicos, 22 pacientes apresentaram melhora após a laminectomia (48\%), 22 permaneceram inalterados (48\%) e houve piora neurológica em um paciente. A Tabela 2 relaciona as diferentes variáveis estudadas com o desenvolvimento de melhora neurológica de acordo com a escala de Frankel. A presença de síndrome de secção medular completa apresentou baixo potencial para recuperação (20\%), enquanto que nos pacientes com lesão de cauda equina a melhora foi observada em $60 \%$ dos casos. O uso de glicocorticóide, o momento cirúrgico e a presença de laceração dural não demonstraram ter significância estatística para a melhora funcional destes pacientes.

Dos 14 pacientes que apresentavam dor à admissão, observou-se que em dez (71\%) houve melhora total ou parcial após a descompressão cirúrgica. A dor persistiu inalterada em três pacientes (21\%) e em apenas um houve piora. Dos nove pacientes que referiam dor radicular à admissão, um persistiu com dor e outro piorou após o procedimento cirúrgico; dos quatro casos referindo dor no local de penetração do projetil, apenas um não obteve alívio com a cirurgia; o paciente com queixa de disestesias em membros inferiores não obteve melhora da dor.

As complicações observadas foram: um caso de hemorragia digestiva devido a sangramento de úlcera duodenal, um caso de infecção de ferida operatória associada a abcesso para-espinhal, sete casos de infecção do trato urinário devido a uso prolongado de sonda vesical. Em um paciente houve piora do déficit motor no pós-operatório. Não foi observado nenhum caso de meningite. Um paciente desenvolveu fístula liquórica após a cirurgia; neste caso não foi possível a síntese hermética da dura-máter . 


\section{DISCUSSÃO}

A laminectomia descompressiva tem sido a principal modalidade cirúrgica indicada para o tratamento de TRM por PAF, apesar da indicação cirúrgica ser ainda motivo de controvérsia ${ }^{4,5,7,8,13}$. Aarabi e col. ${ }^{4}$ concluíram que a cirurgia não melhorou o prognóstico dos pacientes, além de ter aumentado o número de complicações. Já Benzel e $\operatorname{col}^{\prime} .{ }^{1}$ mostraram que até $86 \%$ dos doentes com lesão incompleta obtêm melhora com a descompressão cirúrgica. O serviço de neurocirurgia do HBDF utiliza um esquema terapêutico agressivo para o tratamento deste tipo de lesão: a maioria dos pacientes recebe corticoesteróides segundo esquema já descrito, antibioticoterapia por no mínimo dez dias, tratamento cirúrgico em todos os casos em que se identifica PAF no interior do canal medular, ou ainda (o que é mais comum em relação a coluna cervical) fixação quando se identifica instabilidade. Não são candidatos a cirurgia os casos nos quais o projetil transfixa o canal medular.

O uso de glicocorticóide na maioria de nossos pacientes baseia-se nos estudos multicêntricos para fraturas da coluna realizados nos EUA no início da década de 90 (NASCI II) que provaram o valor da metilprednisolona como fator de melhora funcional destes casos ${ }^{14}$. Artigos publicados posteriormente, porém, sugerem que o uso desta medicação, nos casos de TRM penetrante, não tem o mesmo valor prognóstico ${ }^{15,16}$ descrito para os casos de TRM fechado. Este nosso trabalho confirma os estudos anteriores, pois não encontramos diferença estatística entre o grupo que usou e o grupo que não usou glicocorticóides dentre os pacientes que apresentaram melhora neurológica. Nossa casuística, no entanto, é relativamente pequena. Ainda, os casos de hemorragia digestiva e infecção de ferida encontravam-se no grupo submetido a corticoterapia por até $24 \mathrm{~h}$.

Vários estudos indicam o uso prolongado de antibióticos apenas quando o projetil cruza vísceras ocas antes de atingir a coluna ${ }^{17-19}$. Por outro lado, Heary e col. ${ }^{17}$ sugerem o uso de antibióticos de largo espectro em todos os casos. O índice de infecção para este tipo de lesão (infecção de ferida, abcessos intra ou paravertebrais, discites, osteomielites) encontrado na literatura varia entre sete e $22 \%{ }^{2,17,18}$. A utilização de antibióticos por período prolongado e em todos os casos, o que é a rotina do HBDF, ocorre por considerarmos o ferimento por arma de fogo uma ferida contaminada, principalmente por bactérias da pele como o S. epidermides e $S$. aureus. Nesta série, apenas cinco pacientes apresentavam associação com perfuração de vísceras e nenhum desenvolveu infecção. Nosso índice de infecção foi $2,2 \%$, isto é, um caso de abcesso para-vertebral pós-operatório.

Uma das variáveis mais aceitas pela literatura como fator prognóstico é a apresentação clínica à admissão $0^{2,4,7,9,11}$, ou seja, lesões completas apresentam muito baixo potencial de recuperação, enquanto que lesões incompletas, e, principalmente, lesões de cauda equina, apresentam potencial de recuperação de 47 e $86 \%$, respectivamente. O presente estudo confirma os dados já conhecidos. Observou-se recuperação de $20 \%$ daqueles pacientes com lesões completas, $50 \%$ para os com lesão incompleta e $60 \%$ para os casos com síndrome de cauda equina. Estes números podem explicar porque os doentes com traumas na região lombar apresentam maior potencial de melhora neurológica (favorecendo a indicação cirúrgica destes casos) o que foi observado em 53\% dos pacientes. Mesmo nos casos que se apresentavam em Frankel A, sendo a lesão na coluna lombar, houve recuperação de dois entre os cinco pacientes com ferimentos nesta localização. Já os traumas sobre a região torácica apresentaram o menor índice de recuperação, pois, neste nível, o canal medular é mais estreito, tem o menor suprimento sanguíneo ${ }^{20}$, e, consequentemente, os pacientes apresentam-se mais frequentemente com síndrome de secção completa. Apenas 36\% destes pacientes apresentaram alguma melhora neurológica. A região cervical, apesar de conter um segmento importante da medula, apresenta canal mais alargado e com melhor irrigação ${ }^{20}$. Isto pode justificar o melhor potencial de recuperação funcional observado, pois a metade dos pacientes com trauma sobre a região cervical evoluíram com melhora na escala de Frankel. O menor número de casos nesta região da coluna é explicado, dentre outros motivos, pela proteção parcial que as estruturas da face oferecem à coluna cervical antepondo-se à trajetória do projetil ${ }^{21}$. 
A necessidade absoluta da realização de uma cirurgia de urgência em todos os casos é discutível. Neste estudo não se observou diferença estatística, com relação à melhora neurológica ou ao desenvolvimento de infecção, entre o grupo de pacientes operados com menos de 24 horas em comparação com aqueles submetidos ao procedimento cirúrgico mais tardiamente. Em estudo de 88 pacientes, Cybulski e col. ${ }^{5}$ já haviam mostrado não haver diferença na evolução de pacientes operados com mais de 72 horas em comparação ao grupo com menos de 72 horas, enquanto estudo de Robertson e col. ${ }^{11}$ sugere que nas lesões de cauda equina a cirurgia deve ser feita o mais breve possível. Segundo alguns autores ${ }^{8}$ a cirurgia realizada muito precocemente poderia inclusive piorar o déficit neurológico, devido a manipulação de tecido neural edemaciado e a maior dificuldade de hemostasia. Fica claro, no entanto, que nos pacientes com fístula liquórica e naqueles com déficit neurológico em progressão, a cirurgia não pode ser protelada, devendo ser realizada em caráter de urgência ${ }^{2}$.

Teoricamente, a presença de lesão da dura-máter aumentaria as chances de haver também lesão das estruturas neurais que se encontram no seu interior. Devido a isto foi testada a hipótese desta variável influir no prognóstico destes pacientes. Não encontramos diferença estatística entre os grupos com e sem lesão dural em relação à recuperação neurológica, sendo que o número de pacientes que evoluiu favoravelmente em cada grupo foi similar. Este fato sugere que a dura-máter não oferece proteção eficiente contra a lesão mecânica e térmica do projetil. O paciente que apresentou fístula liquórica no pós-operatório encontrava-se no grupo em que não foi realizada a síntese da dura-máter. Isto vem confirmar a importância da síntese dural, mesmo nos casos de grande dificuldade técnica como nas lacerações localizadas anteriormente à medula.

As lesões por PAF são reconhecidamente causadoras de dor intensa, mesmo em pacientes com lesões completas ${ }^{2,11}$. A origem da dor pode ser por compressão radicular, medular ou da cauda equina, destruição de tecido nervoso, e ainda, por instabilidade da coluna lesada ou lesões de partes moles. Richard e col. ${ }^{22}$ não observaram diferença no controle da dor entre pacientes submetidos a procedimento cirúrgico ou tratados conservadoramente. Em nosso estudo, por outro lado, observouse melhora da dor em dez dos 14 pacientes que apresentavam a queixa no pré-operatório, reforçando o importante papel da laminectomia para estes casos. Houve maior incidência da dor radicular, o que pode ser explicado pelo maior número de lesões a nível de coluna lombar e lesões de cauda equina. A literatura mundial concorda que este tipo dor é aquela que melhor responde à terapia cirúrgica, pois geralmente ocorre por compressão da raíz afetada, e não por destruição desta ${ }^{2}$. A dor no local de penetração do projetil decorre de lesão de partes moles e óssea, e também pode ser aliviada com o procedimento cirúrgico, como foi observado em três dos quatro casos com este tipo de queixa. O alívio e prevenção da dor devem sempre ser considerados como um dos principais objetivos da cirurgia.

Em conclusão, os principais fatores prognósticos em pacientes submetidos a laminectomia para trauma raquimedular por PAF foram o nível da lesão e a apresentação clínico-neurológica préoperatória. Os melhores resultados podem ser esperados nos casos de trauma sobre a região lombar e nos pacientes com síndrome de cauda equina. Fatores como utilização de glicocorticóides, momento cirúrgico e presença de lesão dural parecem não ter importância na evolução destes pacientes. A descompressão cirúrgica foi útil na melhora do quadro doloroso na maioria dos pacientes.

Agradecimentos - Os autores gostariam de agradecer ao Dr. Luiz Augusto Casulari Roxo da Motta pela prestimosa colaboração, tendo feito a revisão dos originais do artigo.

\section{REFERÊNCIAS}

1. Benzel EC, Hadden TA, Colemam JE. Civilian gunshot wounds to spinal cord and cauda equina. Neurosurgery 1987;20:281-285.

2. Cheryl A, Muszynsky KN Surgical management of penetrating injuries to the spine. In Schimidek (ed) Operative neurosurgical techniques. 3.Ed. Philadelphia: Saunders, 1995;1971-1980.

3. Walters RL, Adkins RH, Yakura J, Sie I. Profiles of spinal cord injury and recovery after gunshot injury. Clin Orthop 1991;26:14-21.

4. Aarabi B, Alibaii E, Taghipur M, Kamparkur A. Comparative study of functional recovery for surgically explored and conservatively managed spinal cord missile injuries. Neurosurgery 1996;39:1133-1140. 
5. Cybulski GR, Stone JL, Kant R. Outcome of laminectomy for civilian gunshot injuries of the terminal spinal cord and cauda equina: review of 88 cases. Neurosurgery 1989;24:392-397.

6. O’Donell HD, Oshiro E, Ducker TB. Gunshot wound and spinal cord injury. J Spinal Disord 1991;4:114-116.

7. Stauffer ES, Wood RW, Kelly EG. Gunshot wounds of the spine: the effects of laminectomy. J Bone Joint Surg 1979;61:389-392.

8. Simpson RK, Verger BH, Narayan RK. Treatment of acute penetrating injuries of the spine: a retrospective analysis. J Trauma 1989;29:42-46.

9. Ebrahein NA, Savolaine ER, Jackson WT, Andreshak TG, Rayport M. Magnetic resonance imaging in the evaluating of a gunshot wound to the cervical spine. J Orthop Trauma 1989;3:19-22.

10. Kane T, Capen DA, Waters R, Zigler JE, Adkins R. Spinal cord injury from civilian gunshot wounds: the Rancho experience 1980-1988. J Spinal Disord 1991;4:306-311.

11. Robertson DP, Simpson RK. Penetrating injuries restrited to the cauda equina: a retospective review. Neurosurgery 1992;31:265-269.

12. Frankel HL, Hancoek DO. The value of postural reduction in the inicial management of closed injuries of the spine with paraplegia and tetraplegia. Paraplegia 1969;7:179-192.

13. Walters RL, Adkins RH, Yakura JS, Sie I. Effects of surgery on motor recovery following traumatic spinal cord injury. Spinal Cord 1996;34:188-192.

14. Shepard MJ, Bracken MB, Collins WF. Methylprednisolone or naloxone treatment after acute spinal cord injury: 1 year follow-up data. J Neurosurg 1992;76:23-31.

15. Heary RF, Vaccaro AR, Messa JJ, et al.. Steroids and gunshot wounds to the spine. Neurosurgery 1997;41:576-583.

16. Leavy ML, Gans W, Wijeshinge HS, SooHoo WE, Adkins RH, Stillermam CB. Use of methylprednisolone as na adjunt in the management of patients with penetrating spinal cord injury: outcome analysis. Neurosurgery 1996;39:1141-1148.

17. Heary RF, Vaccaro AR, Mesa JJ, Balderstone RA. Thoracolumbar infections in penetrating injuries to the spine. Orthop Clin North Am 1996;27:69-81.

18. Lin S, Vaccaro AR, Reich SM, Devine M, Colter JM, Reisch S. Low-Velocity gunshot wounds to the spine with an associated transperitoneal injury. J Spinal Disord 1995;8:136-144.

19. Rommanick PC, Smith TK, Kopaniky DR, Oldfield D. Infections about the spine associated with low velocity-missile injury to the abdome. J Bone Joint Surg 1985;67:1195-1201.

20. Zipinick RJ, Scalea TM, Troonski SZ, et al. Hemodynamic responses to penetrating spinal cord injuries. J Trauma 1993;35:578-582.

21. Kupcha PC, An HS, Colter JM. Gunshot wounds to the cervical spine. Spine 1990;15:1058-1063.

22. Richard JS, Stover SL, Jaworski T. Effects of bullet removal on subsequent pain in persons with spinal cord injury secundary to gunshot wound. J Neurosurg 1990;73:401-404. 